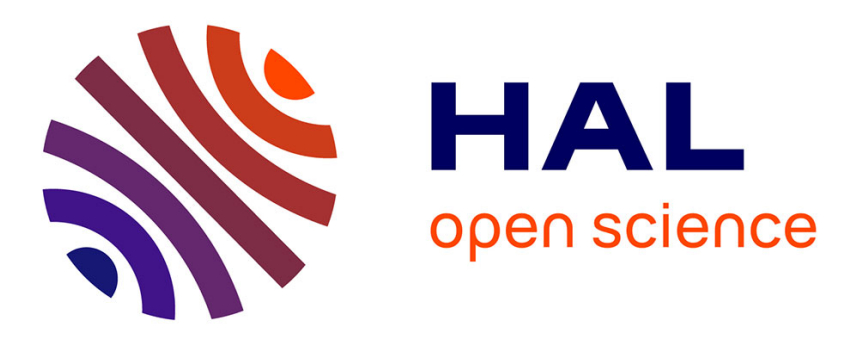

\title{
A Water Solvation Shell Can Transform Gold Metastable Nanoparticles in the Fluxional Regime
}

Chen-Hui Chan, Floriane Poignant, Michael Beuve, Elise Dumont, David Loffreda

\section{- To cite this version:}

Chen-Hui Chan, Floriane Poignant, Michael Beuve, Elise Dumont, David Loffreda. A Water Solvation Shell Can Transform Gold Metastable Nanoparticles in the Fluxional Regime. Journal of Physical Chemistry Letters, 2019, 10, pp.1092-1098. 10.1021/acs.jpclett.8b03822 . hal-02057656

\section{HAL Id: hal-02057656 https://hal.science/hal-02057656}

Submitted on 1 Dec 2020

HAL is a multi-disciplinary open access archive for the deposit and dissemination of scientific research documents, whether they are published or not. The documents may come from teaching and research institutions in France or abroad, or from public or private research centers.
L'archive ouverte pluridisciplinaire HAL, est destinée au dépôt et à la diffusion de documents scientifiques de niveau recherche, publiés ou non, émanant des établissements d'enseignement et de recherche français ou étrangers, des laboratoires publics ou privés. 
This document is confidential and is proprietary to the American Chemical Society and its authors. Do not copy or disclose without written permission. If you have received this item in error, notify the sender and delete all copies.

\section{A Water Solvation Shell can Transform Gold Metastable Nanoparticles in the Fluxional Regime}

\begin{tabular}{|r|l|}
\hline Journal: & The Journal of Physical Chemistry Letters \\
\hline Manuscript ID & jz-2018-038223.R1 \\
\hline Manuscript Type: & Letter \\
\hline Date Submitted by the & nuthor: \\
\hline Complete List of Authors: & $\begin{array}{l}\text { Chan, Chen-Hui; Ecole normale superieure de Lyon, Laboratoire de } \\
\text { Chimie UMR CNRS 5182 } \\
\text { Poignant, Floriane; Universite Claude Bernard Lyon 1, Institut de } \\
\text { Physique Nucléaire de Lyon } \\
\text { Beuve, Michaël; Universite Claude Bernard Lyon 1, Institut de Physique } \\
\text { Nucléaire de Lyon } \\
\text { Dumont, Elise; Ecole normale superieure de Lyon, Laboratory of } \\
\text { Chemistry } \\
\text { Loffreda, David; LABORATOIRE DE CHIMIE, UMR CNRS 5182, ECOLE } \\
\text { NORMALE SUPERIEURE DE LYON }\end{array}$ \\
\hline
\end{tabular}

\section{SCHOLARONE Manuscripts}




\title{
A Water Solvation Shell Can Transform Gold
}

\section{Metastable Nanoparticles In the Fluxional Regime}

\author{
Chen-Hui Chan, $\dagger$ Floriane Poignant,,+ Michaël Beuve,,+ Elise Dumont,$\dagger$ David Loffreda $* \dot{\dagger}$ \\ † Univ Lyon, Ens de Lyon, CNRS UMR 5182, Université Claude Bernard Lyon 1, Laboratoire \\ de Chimie, F-69342 Lyon, France \\ † Univ Lyon, Université Lyon 1, UMR CNRS5822 /IN2P3, IPNL, PRISME, PHABIO, \\ Villeurbanne 69322, France
}

Solvated gold nanoparticles have been modeled in the fluxional regime by density functional theory including dispersion forces for an extensive set of conventional morphologies. The study of isolated adsorption of one water molecule shows that the most stable adsorption forms are similar (corners and edges) whatever the nanoparticle shape and size, although the adsorption strength differs significantly $(0.15 \mathrm{eV})$. When a complete and explicit water solvation shell interacts with gold nanoclusters, metastable in vacuum and presenting a predominance of (100) square facets (ino-decahedra $\mathrm{Au}_{55}$ and $\mathrm{Au}_{147}$ ), these nanoparticles are found unstable and transform into closest morphologies exhibiting mainly (111) triangular facets and symmetries. The corresponding adsorption strength per water molecule becomes independent from shape and size and is enhanced by the formation of two hydrogen-bonds in average. For applications in radiotherapy, this study suggests that the shapes of small gold nanoparticles should be homogenized by interacting with the biological environment. 


\section{TOC GRAPHICS}

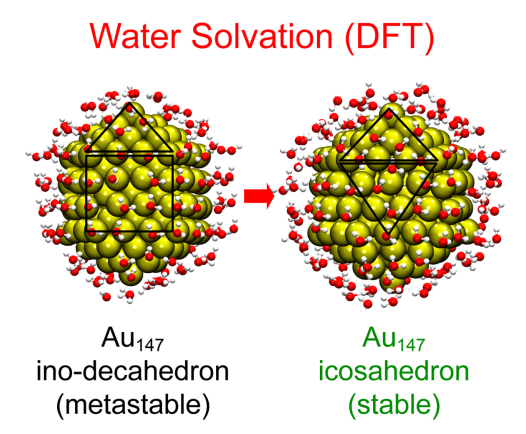

KEYWORDS Density functional theory, Dispersion forces, Gold, Nanoparticles, Water, Solvation.

Functionalized gold nanoparticles (NPs) have been considered in the recent years as promising materials for applications in biomedicine, especially for radiotherapy and cancer treatment. ${ }^{1,2,3,4,5}$ Their excellent biocompatibility, long blood circulation time and the possibility of their functionalization make them highly desirable for theranostics. In fact, they contribute to radiosensitization effects making tumor cells more responsive to ionizing radiation. ${ }^{6,7}$ Under irradiation, Au NPs in water generate more electrons and reactive oxygen species, which may amplify the damages caused by radiation. ${ }^{7,8}$ To date, although the structure of gold nanoparticles has been examined in model conditions by diffraction and microscopic measurements, $,{ }^{9}, 10,11,12,13,14$ little is known at the atomic scale regarding the morphology, structure, energetics of Au NPs in contact with liquid water (biological natural environment).

The lack of information and understanding opens the way to theoretical modeling. Indeed, typical $\mathrm{Au}$ NP working sizes are in the range 30-50 nm. ${ }^{1,3}$ However, smaller Au NPs (1.9 nm) are proposed in the context of radiotherapy enhancement, ${ }^{15,16}$ although monodisperse ultra-small NPs 
are difficult to synthesize. Hence theoretical simulations of Au NPs approaching this range can bring an interesting understanding. For instance, interfacial chemical and physical processes happening at the vicinity of functionalized Au NPs can be explored by atomistic calculations while water radiolysis can be studied by continuous models and Monte Carlo simulations like LiQuiD code. ${ }^{17,18}$. These two complementary approaches may provide a better understanding of interfacial properties while Monte Carlo simulations may benefit from useful atomistic information. Concerning the modeling of the interface between Au NPs and water solvation at the atomic scale, Density Functional Theory (DFT) is a powerful tool to predict optimal geometries and energetics. In DFT studies, static approaches are often considered to describe water adsorption on $\mathrm{Au}$ surfaces ${ }^{19,20,21,22,23,24}$ and nanoclusters up to 20 atoms, ${ }^{25}$ although the explicit interface between metallic nanoparticles and liquid water can be described by ab initio molecular dynamics simulations. ${ }^{26} \mathrm{Up}$ to date, a few studies based on parametrized force fields and classical molecular dynamics simulations have been reported in the literature for gold/water interfaces. ${ }^{27,28,23}$ Regarding the question of Au NPs most likely morphologies and shapes as a function of size and temperature, a few investigations have been proposed based on either Au NPs modeled in vacuum by explicit 3D structures $29,30,31,32,33,34$ or Au NPs in water vapor environment described by extended metallic surfaces calculated by DFT and Wulff construction for predicting thermal properties. ${ }^{35}$ According to a recent DFT study, ${ }^{32} \mathrm{Au}$ NPs in vacuum can exist in various shapes at different size in the fluxional regime [1-3.5 nm] with a preference for icosahedral morphology (decahedral and octahedral ones being metastable). Global optimization methods based on semi-empirical potentials have shown the versatility of gold shapes in the fluxional regime (below 100 atoms). ${ }^{29,30}$ At a larger scale (1000 atoms), these approaches show that truncated octahedra and Marksdecahedra are in competition, whereas icosahedra are the least stable NPs. ${ }^{31,36,37,38}$ In addition, 
cuboctahedra and ino-decahedra become more stable than icosahedra ${ }^{31}$ in contrast with a recent DFT study at large NP size. ${ }^{32}$ Water monomer on small gold nanoclusters (less than 20 atoms) mainly interacts with gold at corner adsorption sites with a variable stability, depending also on the choice of the DFT functional (0.2-0.4 eV with vdW-DF and 0.25-0.5 eV with PBE). ${ }^{25}$ This stability is larger than the adsorption energy calculated on $\mathrm{Au}(111)(-0.105 /-0.14 \mathrm{eV}$ with PW91 ${ }^{19,20},-0.11 \mathrm{eV}$ with PBE, ${ }^{21,22}-0.24 \mathrm{eV}$ with PBE-D2, ${ }^{21}-0.30 \mathrm{eV}$ with optB86b-vdW, ${ }^{21}$ and $0.192 \mathrm{eV}$ with revPBE-vdW ${ }^{22}$ ). The water vapor effect on Au nanoparticle shape is rather small by comparison with other metals, and consists in a progressive transformation of (100) facets in favor of (111) ones becoming larger in truncated octahedra. ${ }^{35}$

Hence, an explicit static or dynamic atomistic model describing the interaction of water monomers and solvation shells (in particular first solvation shell around the nanoparticle) with gold nanoparticles in the range 1-2 $\mathrm{nm}$ is still missing. In this work, we develop DFT models of water adsorption on a family of several convex and regular gold polyhedra (including octahedral and icosahedral shapes), and of water solvated Au NPs with a static approach including dispersion forces. We aim to probe the water effect on Au NPs morphology and size as a first model describing the influence of the biological environment. In particular, we examine, in a comprehensive and systematic study, the adsorption energetics for water especially for decahedral gold shapes, a competitive form which has been rarely considered in the theoretical literature at the DFT level so far.

The first step is the determination of competitive morphologies in the range of sizes accessible by DFT calculations. In Figure 1, the polyhedra considered in this study are defined including the three key octahedral, icosahedral and decahedral symmetries according to the literature. ${ }^{36,37}$ See Figures S1-S6 for all the optimal structures of the considered 44 NPs. 


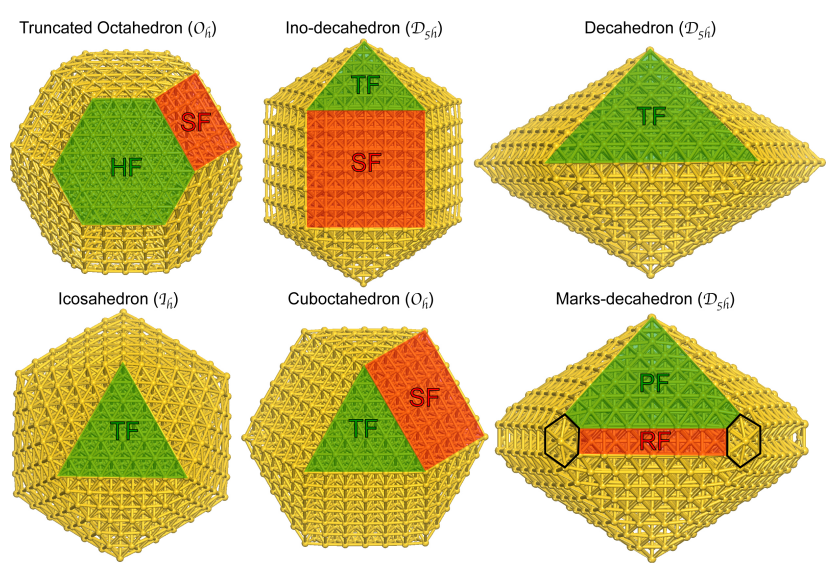

Figure 1. Definition of the considered Au NP morphologies in the range 0.9-3.4 nm (44 NPs): truncated octahedra (to), cuboctahedra (cubo), ino-decahedra (ino) and Marks decahedra (marks) are composed of mixed facets (hexagonal HF, triangular TF, pentagonal PF with square SF or rectangular RF facets), whereas decahedra (deca) and icosahedra (ico) presents only triangular facets (TF).

The question of the most stable morphology according to the size of the NP is a debate. ${ }^{31,32,36,37,38}$ In this work we address this question by DFT calculations including dispersion forces. Figure 2a plots the cohesion energy of the NP in vacuum against $\mathrm{N}^{-1 / 3}$ (see Eq. S1 in the Supporting Information). For each polyhedral family among the 6 key shapes exposed in Figure 1, we have quantified the slopes and the offsets of the linear laws obtained in the range 0.9-3.4 nm (38-976 atoms). In addition, we propose to compare the trends coming from the cohesion energy with those resulting from the calculation of excess energy (normalized by $\mathrm{N}^{-2 / 3}$, see Eq. S2 in the Supporting Information), as displayed in Figure $2 b$, a relevant descriptor which separates more clearly the nanoparticle stability. ${ }^{36,37}$. In Figure $2 \mathrm{a}$, the linear laws for the cohesion energy show that two terms are in competition for the NP stability: the offset corresponding to the cohesion energy in the Au bulk and the slope related to the average facets surface energy including facet, edge and 
corner atoms. The obtained offsets are close to the calculated bulk cohesion energy ( $-3.695 \mathrm{eV} / \mathrm{at}$.) in good agreement with experiment (-3.81 eV/at.). Our linear models are in better agreement with experiments than the previous DFT work $^{32}(-3.285 \mathrm{eV}$ for the icosahedra with the TPSS functional). However, they differ slightly from one polyhedral family to another one since the examined range of NP size is limited (below $3.4 \mathrm{~nm}$ ). The truncated octahedral NPs have the most stable offset (-3.736 eV/at.). They compete with icosahedra and Marks-decahedra with respective bulk cohesion energy of $-3.714 \mathrm{eV} /$ at. and $-3.711 \mathrm{eV} /$ at. At the opposite, the decahedral NPs (regular and Marks) show the minimal facet surface energies $(3.367 \mathrm{eV})$. This is expected since these polyhedra exhibit mainly large (111)-facet type. Then comes the icosahedra with small (111)-facet type (3.428 eV in this work whereas $2.478 \mathrm{eV}$ was proposed previously ${ }^{32}$ ). The NPs having the highest average facet surface energy are truncated octahedra, ino-decahedra and cuboctahedra since they possess large (100)-facet type, well known to be less stable than (111) type. In order to clear this picture, we have examined the NP relative stability with the excess energy. In Figure $2 b$, the calculations show that two shapes compete in the range 0.9-1.8 nm : truncated octahedral and decahedral (regular and Marks); $\mathrm{Au}_{55}$ icosahedron being also competitive. Above $1.8 \mathrm{~nm}$ (201 atoms), the truncated octahedra become really more stable than the other polyhedra. At $3.4 \mathrm{~nm}$ (about 1000 atoms), they predominate while the Marks-decahedra and icosahedra are minority. Our picture differs from previous systematic studies based on global optimization methods, effective semi-empirical potentials and Monte Carlo simulations concluding that decahedral shapes are either majority or equivalent in stability to truncated octahedral forms. ${ }^{36,37,31,38}$ In addition, the stability of icosahedra tends to slightly increase with the NP size, in contrast with these previous studies. Our global trends are in better agreement with a previous study based on simulations obtained with Sutton-Chen potential ${ }^{39}$ and they also support 
in part the recent DFT study examining a set of Au NPs with the TPSS functional. ${ }^{32}$ Several experimental studies based on HAADF-STEM measurements have been reported for the determination of the structure of gold nanoclusters especially for particular sizes: $309,{ }^{12} 561,{ }^{13}$ and 923 atoms. $^{14}$

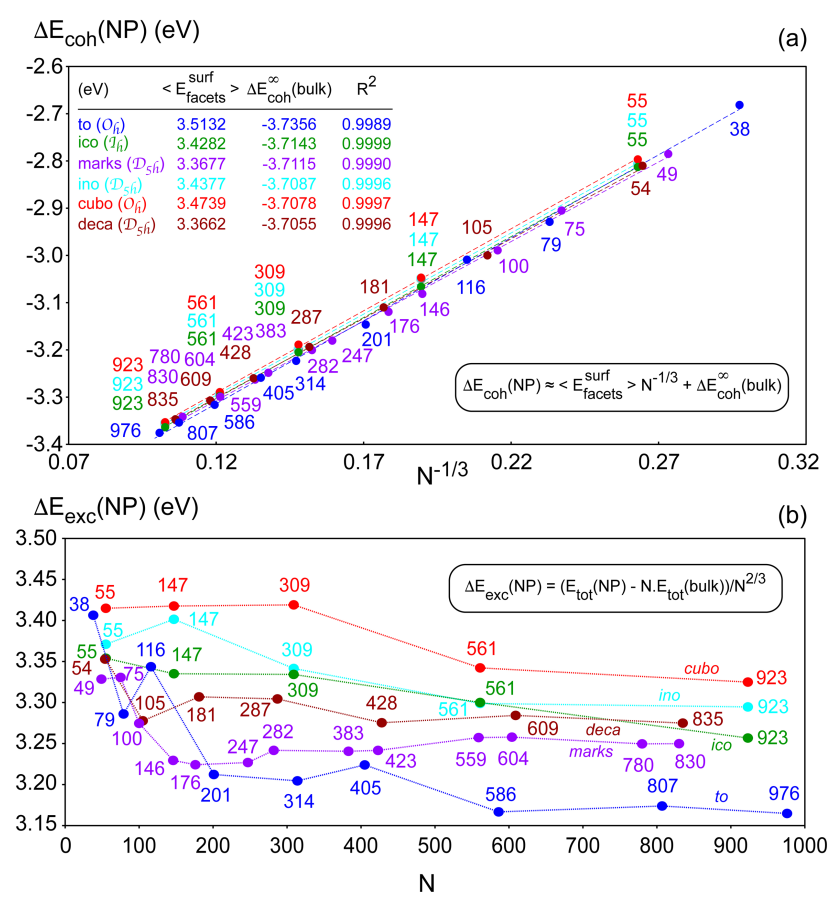

Figure 2. (a) Normalized cohesion energy per atom $\Delta \mathrm{E}_{\mathrm{coh}}(\mathrm{NP})(\mathrm{eV})$ diagram against $\mathrm{N}^{-1 / 3}$, where $\mathrm{N}$ is the number of $\mathrm{Au}$ atoms in the NP. The parameters (slope, offset and $\mathrm{R}^{2}$ ) of the linear regressions are defined in the equation, where the slope $<\mathrm{E}_{\text {facets }}$ surf $>(\mathrm{eV})$ can be identified as the average facet surface energy of the NP (including center, edge and corner atoms) and the offset $\Delta \mathrm{E}^{\infty}$ coh (bulk) (eV) as the cohesion energy extrapolated for the bulk (infinite value of $\mathrm{N}$ ). The six different families of the NPs are reported with different colors defined in the inset where the parameters of the linear regression for each shape family are exposed (the stability for each family being captured in average by the values of the offsets). (b) Excess energy $\Delta \mathrm{E}_{\mathrm{exc}}(\mathrm{NP})(\mathrm{eV})$ diagram against the number $\mathrm{N}$ of $\mathrm{Au}$ atoms in the NP according to the defined formula. 
The reported images in these publications show the preference of defective decahedral and face centered cubic forms with respect to icosahedral shape. Our DFT results support these conclusions since truncated octahedral and Marks-decahedral forms are found more stable than icosahedral shape above 300 atoms.

Based on this study, we have then examined the adsorption properties of a water monomer on the most competitive polyhedra in the range $0.9-1.8 \mathrm{~nm}$ as shown in Figure 3. The truncated octahedra $\mathrm{Au}_{38}, \mathrm{Au}_{79}$ and $\mathrm{Au}_{201}$ have been selected, as well as decahedra $\mathrm{Au}_{54}$ and $\mathrm{Au}_{105}$. In addition, metastable shapes such as icosahedra and ino-decahedra have also been considered in order to explore the impact of water adsorption on their metastability. Due to the complexity of the water solvation shell examined later on, the largest considered cluster is $\mathrm{Au}_{201}$. In Figure 3, for each of the 9 nanoclusters, the most stable adsorption structures have been reported (including adsorption energetics - see Eq. S3 in the Supporting Information, Au-O distance and site), while all the metastable adsorption forms have been presented in Figures S7-S15. Moreover, the decomposition of adsorption energetics in covalence and dispersion contributions is exposed in Table S1. As the size of the NP increases, the adsorption site changes (although the adsorption at a corner remains majority) and the evolution of the adsorption energy is not monotonous with values in a narrow range: from -0.31 to $-0.46 \mathrm{eV}$. Our results agree with previous DFT values proposed on Au small clusters $^{25}$ and $\mathrm{Au}(111) \cdot{ }^{21,22}$ The adsorption of a water molecule is thus a weak chemisorption with a quite long Au-O distance in the range 2.40-2.63 $\AA$. This is consistent with the weak deformation of $\mathrm{Au}$ NPs after adsorption. In order to quantify this, an energy decomposition analysis of adsorption energetics is proposed in Figure 4. 

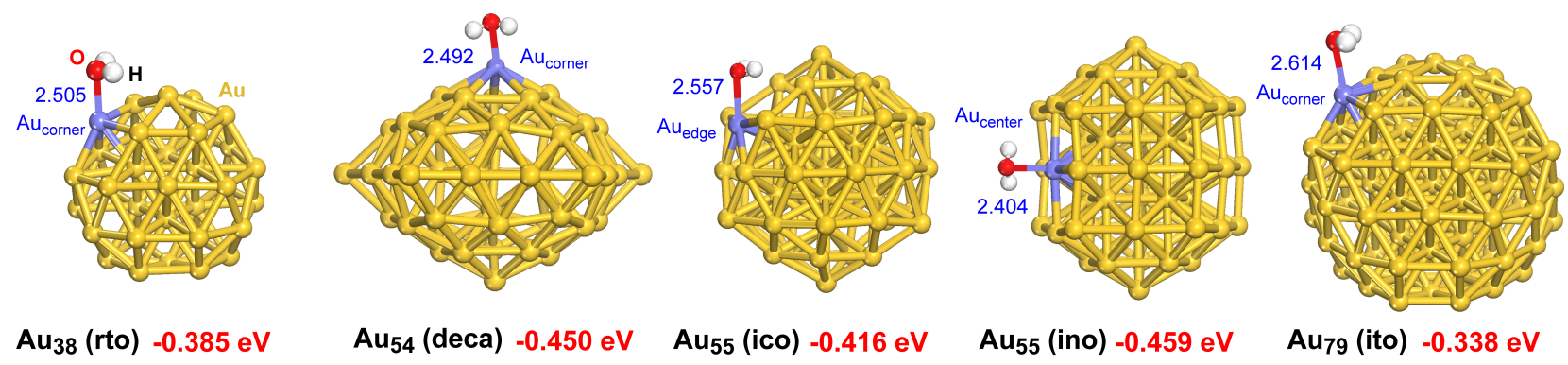

$\mathrm{Au}_{54}$ (deca) $-0.450 \mathrm{eV}$

$\mathrm{Au}_{55}$ (ico) $-0.416 \mathrm{eV}$

$\mathrm{Au}_{55}$ (ino) $-0.459 \mathrm{eV}$

$\mathrm{Au}_{79}$ (ito) $-0.338 \mathrm{eV}$

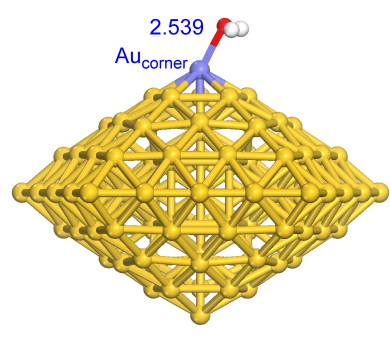

$\mathrm{Au}_{105}$ (deca) $-0.387 \mathrm{eV}$

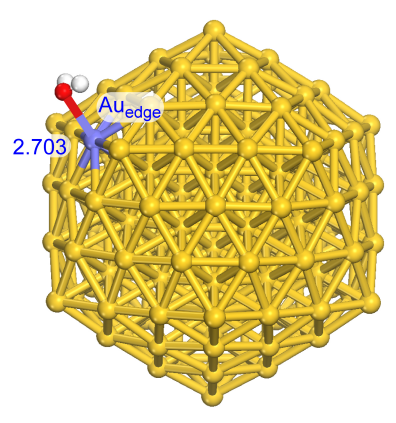

$\mathrm{Au}_{147}$ (ico) $-0.312 \mathrm{eV}$

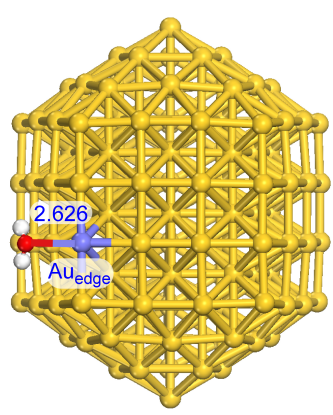

$\mathrm{Au}_{147}$ (ino) $-0.338 \mathrm{eV}$

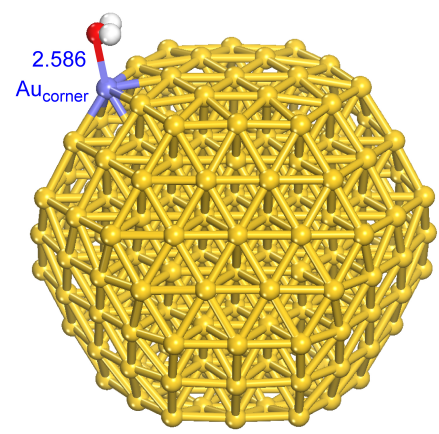

$\mathrm{Au}_{201}$ (rto) $-0.346 \mathrm{eV}$

Figure 3. Optimized adsorption structures of a water monomer on nine different Au NPs in the range 0.9-1.8 nm, including four competitive morphologies : regular and irregular truncated octahedron (rto and ito, respectively), icosahedron (ico), ino-decahedron (ino) and decahedron (deca). The definitions of colors for atoms are given, as well as the location of the surface Au adsorption site with the following possibilities: corner, edge, facet center. The Au-O bond distances are reported in $\AA$ (blue characters), whereas the adsorption energies in $\mathrm{eV}$ (red characters).

According to this analysis, the deformation energy of the Au NPs is positive and weak in general for all the shapes, in the range $0.01-0.14 \mathrm{eV}$. In parallel, the deformation energy of water monomer is also positive and close to zero (below $8 \mathrm{meV}$ ). This means that the non-monotonous trend of adsorption energy is mainly captured by the interaction energy between the Au cluster and water. Moreover, this interaction which corresponds to the chemical bonding between both partners is the strongest one $(-0.6 \mathrm{eV})$ for the ino-decahedral NPs and for the icosahedral shape at least for 
small size $(-0.47 \mathrm{eV})$. For the most stable NPs (truncated octahedra and decahedra), the chemical bonding is quite weaker (from -0.32 to $-0.47 \mathrm{eV}$ ) and it varies less with the cluster size.

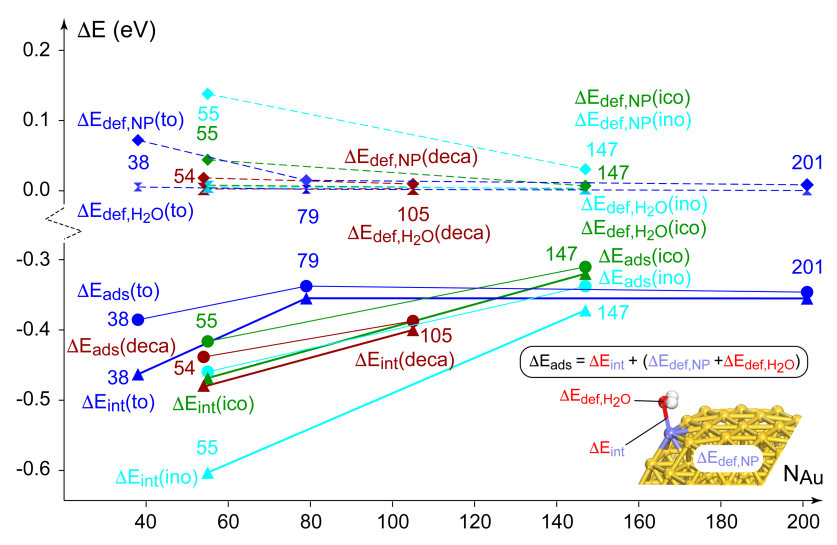

Figure 4. Energy diagram against the number of Au atoms in the NPs, showing the energy decomposition analysis (EDA) of the adsorption energy $\left(\Delta \mathrm{E}_{\mathrm{ads}}, \mathrm{eV}\right)$ of water monomer on nine different Au clusters with four different shapes (defined by specific colors) in the range 0.9-1.8 $\mathrm{nm}$ (see Figure 2 for the optimal adsorption structures). $\Delta \mathrm{E}_{\text {ads }}$ is expressed in the equation as the sum of the pure binding energy between $\mathrm{Au} N \mathrm{NP}$ and water ( $\Delta \mathrm{E}_{\mathrm{int}}$, energy gain), the deformation energy of water between optimal isolated situation and the geometry in the adsorption form $\left(\Delta \mathrm{E}_{\mathrm{def}, \mathrm{H} 2 \mathrm{O}}\right.$, energy cost) and the equivament deformation energy of $\mathrm{Au} \mathrm{NP}\left(\Delta \mathrm{E}_{\mathrm{def}, \mathrm{NP}}\right.$, energy cost).

On the basis of the best adsorption structures of water monomers on the Au NPs, we then propose to explore the interaction of a complete shell of water molecules around the metallic clusters by a static approach. The starting geometries of these solvation shells come from an additive principle assuming that the coadsorption of the best sites is an optimal choice for maximizing the overall stability of the solvated nanocluster. This means that, for the various NPs, all the equivalent sites corresponding to the best adsorption form of monomers are occupied by water molecules. Then, to complete the solvation shells, metastable adsorption sites were considered in such a way that a 
network of flat cophysisorbed and cochemisorbed water molecules is built up through hydrogen bonds with reasonable distances (longer than $1.8 \AA$ ). Thus, since all the molecules were initially coplanar with respect to one facet, no arbitrary choice related to H-up/H-down configuration has been considered by construction. This starting choice was also guided by a previous molecular dynamics study of immersed Au clusters (13, 55 and 147) in water, showing that the hydrogen bonding of water molecules is arranged in a two-dimensional structure for $\mathrm{Au}_{55}$ and $\mathrm{Au}_{147 .}{ }^{28} \mathrm{The}$ final structures of the solvation shells obtained after geometry optimizations are reported in Figure 5. Details of the total number of water molecules per NP, the numbers of chemisorbed and physisorbed molecules, and analyses of adsorption energetics, nanoparticle structural deformation induced by water monoshells and hydrogen bonds are given in Tables S2, S3 and Figure S17. The surface coverage of water is addressed in Figure 5, with the ratio of chemisorbed and physisorbed molecules. When the size of the NP increases, this coverage decreases in average progressively from 0.44 to $0.23 \mathrm{ML}$ with two exceptions at sizes 55 and 147 for icosahedral $(0.40 \mathrm{ML})$ and inodecahedral $(0.31 \mathrm{ML})$ shapes. In a concomitant way, the number of chemisorbed molecules decreases in favor of physisorbed water (the ratio decreasing in average from 0.27 to 0.15 ), again with two exceptions for $\mathrm{Au}_{55}(0.28)$ and $\mathrm{Au}_{147}$ (0.22). At the largest size $\left(\mathrm{Au}_{201}\right)$, the calculated coverage of $0.23 \mathrm{ML}$ is smaller than the one proposed previously $(0.29 \mathrm{ML})$ for an explicit liquid water/ $\mathrm{Pt}_{201}$ interface investigated by ab initio molecular dynamics at $350 \mathrm{~K} .{ }^{26}$ Our result for $\mathrm{Au}$ with a static approach is thus reasonable since the chemical bonding on $\mathrm{Au}_{201}(-0.35 \mathrm{eV})$ is almost twice weaker than the one found on $\mathrm{Pt}_{201}(-0.54 \mathrm{eV})$. For the two exceptions of larger coverage of chemisorbed water on $\mathrm{Au}_{55}$ and $\mathrm{Au}_{147}$ (icosahedra and ino-decahedra), this is only a direct consequence of the stronger interaction energy or chemical bonding discussed before, not the fact that these clusters would present more stable adsorption sites in number. 
At first glance, these optimal structures are relatively homogeneous regarding the distribution of chemisorbed and physisorbed water and as a function of the NP size; although no clear arrangement appears in agreement with a previous MD study. ${ }^{28}$ In addition, the solvation shells are quite spherical around the Au NPs, thus meaning that the average interaction between each water molecule and the cluster should not vary much. This can be seen with the average adsorption energy per water (from -0.607 to $-0.643 \mathrm{eV} /$ water) which does not change much against the nanocluster size (see Eq. S4 in the Supporting Information). This contrasts with the adsorption of the monomer discussed before. The adsorption energy per water is in average $70 \%$ stronger for the solvation shell with respect to the isolated adsorption. According to the optimal geometries, this gain is certainly not due to a strengthening of chemisorbed water but rather to the formation of chemical bonds between the molecules (hydrogen bonds). In fact, on the basis of the energetic models presented in the Supporting Information (see Eqs. S7-S13, Table S3 and Figure S18), the adsorption energy per water of the solvation shell is mainly due to the formation of two hydrogen bonds in average (from -0.49 to $-0.59 \mathrm{eV} /$ water depending on the NP size), the rest being the interaction energy between water and gold (around $-0.1 \mathrm{eV} /$ water). In the case of Au NPs, the formation of the water shell occurs at the detriment of chemisorption, since the latter is rather weak. Hence, for Au NPs in the range $0.8-1.9 \mathrm{~nm}$, both the stability of the solvation shell $(-0.6$ $\mathrm{eV} /$ water) and the average number of formed hydrogen bonds (golden rule of 2) are remarkably independent from the NP size and morphology. By comparison, in bulk water, the cohesion energy per water molecule is known from experiments $(-9.9 \mathrm{kcal} . \mathrm{mol}-1$ or $-0.429 \mathrm{eV} /$ water $) .{ }^{40} \mathrm{In}$ addition, the two-body contribution of the dissociation energy of water dimers benchmarked by coupled cluster calculations on configurations extracted from ab initio molecular dynamics simulations of liquid water has been evaluated to $-0.137 \mathrm{eV}$ (while it is $-0.221 \mathrm{eV}$ at equilibrium in gas phase). ${ }^{41}$ 
This means that the maximum number of hydrogen bonds per water molecule in bulk liquid can be estimated to 3.13 bonds, considering that only hydrogen bonding contribute to the cohesion energy in the liquid. This assumption agrees with previous experimental measurements from neutron diffraction ${ }^{42}$ (3.58 hydrogen bonds per water molecule) and with a Car-Parrinello molecular dynamics simulation study ${ }^{43}$ (3.48 hydrogen bonds per water molecule). Hence, we found a decrease of the number of hydrogen bonds in our solvation shells ("onion peel") around Au NPs (from 2.06 to 2.51 hydrogen bonds per water, see Table S3) by comparison with bulk water, in fair agreement with a previous MD study. ${ }^{27}$

Looking at now the impact of the water solvation shells on the geometry of the Au NPs after the geometry optimizations, deformations of the clusters are observed in Figure 5, even for the most stable case in vacuum such as $\mathrm{Au}_{38}, \mathrm{Au}_{54}, \mathrm{Au}_{79}$. This impact is also seen for the less stable icosahedra $\mathrm{Au}_{55}$ and $\mathrm{Au}_{147}$. A quantitative analysis of the structural deformation of the nanoparticle outershells upon water adsorption is addressed in the Supporting Information (see Eqs. S5-S6, Table S2 and Figure S17). Moreover, the metastable ino-decahedra $\mathrm{Au}_{55}$ and $\mathrm{Au}_{147}$ in vacuum are transformed into corresponding icosahedra through the interaction with the water shells. This original result is counterintuitive since water chemisorption on $\mathrm{Au}$ is quite weak as exposed before and HAADF-STEM images show that gold icosahedral nanoclusters in model operating conditions (in the range 1-3 nm) transform into decahedral structures. ${ }^{12,13,14}$ However, the increase of the chemical bond with water obtained for these clusters and the concomitant increase of chemisorbed water coverage discussed previously allow us to explain the origins of such a remarkable phenomenon. Then in this study we demonstrate that water, standing for a model of the biological environment, may have a significant impact on the morphology of metastable NP shapes, although the intrinsic interaction with water is relatively weak. 

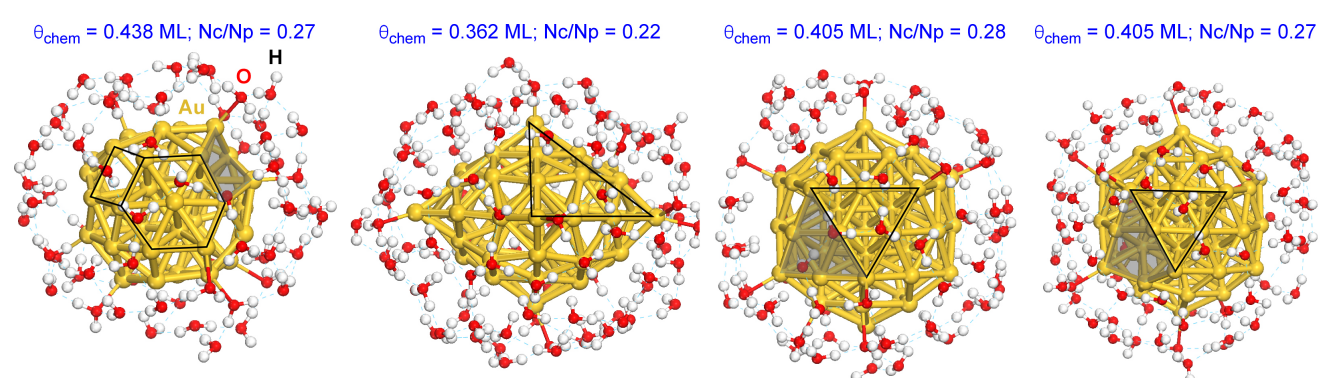

$\theta_{\text {chem }}=0.317 \mathrm{ML} ; \mathrm{Nc} / \mathrm{Np}=0.21$

$\mathrm{Au}_{38}$ (rto) $-0.608 \mathrm{eV} / \mathrm{w}$.

$\mathrm{Au}_{54}$ (deca) $-0.639 \mathrm{eV} / \mathrm{w}$.

$\mathrm{Au}_{55}$ (ico) $-0.611 \mathrm{eV} / \mathrm{w}$.

$\mathrm{Au}_{55}$ (ino) $-0.607 \mathrm{eV} / \mathrm{w}$.
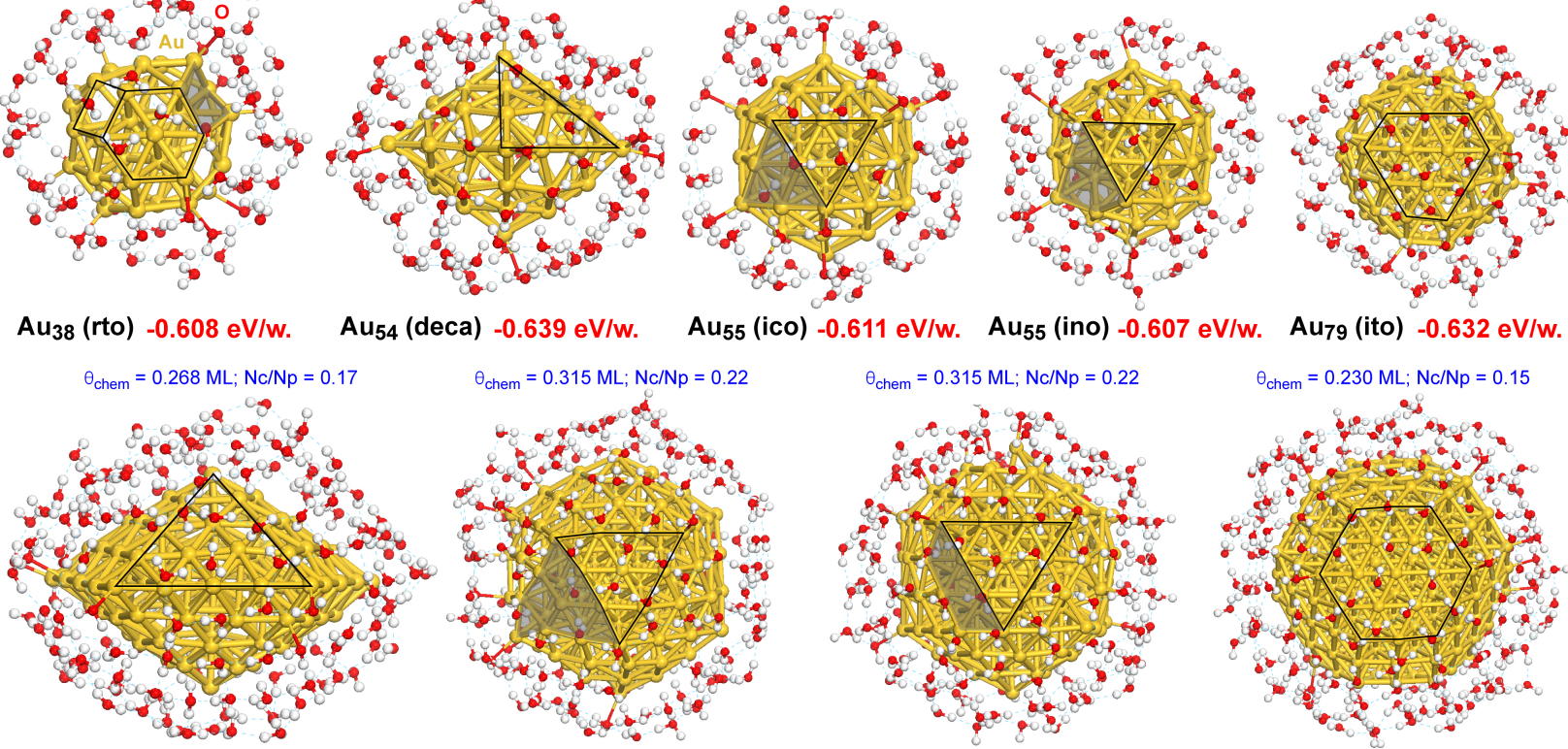

$\mathrm{Au}_{105}$ (deca) $-0.623 \mathrm{eV} / \mathrm{w}$.

$\mathrm{Au}_{147}$ (ico) $-0.643 \mathrm{eV} / \mathrm{w}$.

$\mathrm{Au}_{147}$ (ino) $-0.627 \mathrm{eV} / \mathrm{w}$.

$\mathrm{Au}_{201}$ (rto) $-0.642 \mathrm{eV} / \mathrm{w}$.

Figure 5. Optimized adsorption structures of a shell of water molecules on nine different Au NPs in the range $0.9-1.8 \mathrm{~nm}$, including four competitive morphologies: regular and irregular truncated octahedron (rto and ito, respectively), icosahedron (ico), ino-decahedron (ino) and decahedron (deca). The definitions of colors for atoms are given. The adsorption energies in eV per water molecule in total are reported (red characters), as well as the surface coverage of chemisorbed water molecules in ML (monolayer) and the ratio $\mathrm{Nc} / \mathrm{Np}$ between chemisorbed water $(\mathrm{Nc})$ and physisorbed water $(\mathrm{Np})$. The complex network of hydrogen bonds is also marked by light gray dotted lines. The strongly distorted facets of icosahedral (and transformed ino-decahedral) shapes are drawn with full gray areas for $\mathrm{Au}_{55}$ and $\mathrm{Au}_{147}$

Our DFT works open promising perspectives for a re-parametrization of semi-empirical potentials and global optimization methods aiming to describe the relative stability between ino-decahedra and icosahedra in interaction with a water solvation shell at larger NP size. Especially this would allow the study of $\mathrm{Au}_{309}, \mathrm{Au}_{561}$ and $\mathrm{Au}_{923}$, both with static and dynamic approaches for $\mathrm{Au} /$ water 
interfaces and this would show whether the water solvation still induces the transformation between metastable morphologies at larger NP size.

\section{COMPUTATIONAL METHODS}

DFT calculations (spin restricted) of Au NPs and water adsorption were performed by using the VASP code, version 5.3.5. ${ }^{44,45,46} \mathrm{PBE}^{47}$ with Grimme's D3 semi-empirical dispersion corrected functional $^{48}$ (zero-damping formalism) was considered to describe electronic exchange and correlation at the generalized gradient approximation with van der Waals interactions. The coreelectrons were described by the projector-augmented wave ${ }^{49}$ (PAW) pseudo-potentials (11 valence electrons per $\mathrm{Au}$ atom), and valence electrons were expanded in plane waves with a kinetic cutoff energy of $400 \mathrm{eV}$. All the Au NPs were modeled in a cubic box of $5 \times 5 \times 5 \mathrm{~nm}^{3}$ with a $\Gamma$-point only approach for the k-point mesh and related Brillouin zone. For the Au bulk, the fcc crystalline structure was described by using a k-point grid of $17 \times 17 \times 17$. In the geometry optimizations, the $\mathrm{Au}$ NPs and water adsorption structures were relaxed completely with $10^{-6} \mathrm{eV}$ for the convergence of the total electronic energy and $-0.01 \mathrm{eV} . \AA^{-1}$ for the minimization of the residual forces on the nuclei. A Methfessel-Paxton smearing was used for the calculation of the total electronic energy.

\section{ASSOCIATED CONTENT}

\section{Supporting Information}

- Definitions of Au NP cohesion energy, excess energy, of adsorption energy for water monomers and monoshells

- Figures S1-S6 : complete set of optimized structures of free Au NPs (44 NPs for 6 different morphologies) 
- Figure S7-S15 : complete set of optimized adsorption structures of water monomers on $9 \mathrm{Au}$ NPs in the range 0.9-1.8 nm (adsorption sites and energies, Au-O distances).

- Figure S16 : adsorption energy decomposition model in covalence and dispersion for water monomers on Au NPs in the range $0.9-1.8 \mathrm{~nm}$

- Table S1 : adsorption energy decomposition model in covalence and dispersion for water monoshells on Au NPs in the range 0.9-1.8 nm

- Equations S1-S13 : the definitions of excess energy, cohesion energy for free Au NPs, adsorption energy for water, hydrogen bonding model and roundness degree are all addressed.

- Table S2 and Figure S17 : geometric analysis of the nanoparticle deformation upon water adsorption based on the variation of the roundness degree.

- Table S3 and Figure S18 : hydrogen bonding model for water shells on Au NPs (9 nanoclusters, number of chemisorbed and physisorbed water molecules, adsorption energy of best site for monomers, normalized adsorption energy per water for shells, total hydrogen bonding energy per water and number of hydrogen bonds per water in the shells).

The supporting information is available free of charge on the ACS Publications website at DOI:.

\section{AUTHOR INFORMATION}

\section{Corresponding Author}

*D. L.: E-mail: david.loffreda@ens-lyon.fr ORCID: 0000-0001-9912-7965

\section{Notes}

The authors declare no competing financial interests. 


\section{ACKNOWLEDGMENT}

The authors thank Dr Christine Mottet (CiNAM, Marseille, France) for helpful discussions. They also thank IDRIS in Paris, CINES in Montpellier, TGCC in Grenoble (project 609, GENCI/CT8) and PSMN in Lyon for CPU time and assistance. Chen-Hui Chan thanks LABEX PRIMES (ANR11-LABX-0063) of Université de Lyon (within the program "Investissements d'Avenir" ANR-11IDEX-0007) for the PhD funding. Floriane Poignant thanks «Fondation ARC pour la recherche sur le cancer » for the support. The authors thank the SYSPROD project and AXELERA Pôle de Compétitivité for financial support (PSMN Data Center).

\section{REFERENCES}

(1) Laprise-Pelletier, M.; Simao, T. ; Fortin, M.-A. Gold nanoparticles in radiotherapy and recent progress in nanobrachytherapy. Adv. Healthcare Mater. 2018, $\mathrm{n}^{\circ} 1701460-1,27$, DOI: 10.1002/adhm.201701460.

(2) Henderson, L.; Neumann, O.; Kaffes, C.; Zhang, R.; Marangoni, V.; Ravoori, M. K.; Kundra, V.; Bankson, J.; Nordlander, P.; Halas, N. J. Routes to potentially safer T1 magnetic resonance imaging contrast in a compact plasmonic nanoparticle with enhanced fluorescence. $A C S$ Nano 2018, 12 (8), 8214-8223, DOI: 10.1021/acsnano.8b03368.

(3) Gilles, M.; Brun, E.; Sicard-Roselli, C. Gold nanoparticles functionalization notably decreases radiosensitization through hydroxyl radical production under ionizing radiation, Colloids and Surfaces B: Biointerfaces 2014, 123, 770-777, DOI: 10.1016/j.colsurfb.2014.10.028.

(4) Brun, E.; Sicard-Roselli, C. Actual questions raised by nanoparticle radiosensitization. Radiation Physics and Chemistry 2016, 128, 134-142. DOI: 10.1016/j.radphyschem.2016.05.024. 
(5) Her, S.; Jaffray, D. A.; Allen, C. Gold nanoparticles for applications in cancer radiotherapy: Mechanisms and recent advancements. Advanced Drug Delivery Reviews 2017, 109, 84-101. DOI: 10.1016/j.addr.2015.12.012.

(6) Kobayashi, K.; Usami, N.; Porcel, E.; Lacombe, S.; Le Sech, C. Enhancement of radiation effect by heavy elements. Mutation Research 2010, 704 (1-3), 123-131, DOI: 10.1016/j.mrrev.2010.01.002

(7) Peukert, D.; Kempson, I.; Douglass, M.; Bezak, E. Metallic nanoparticle radiosensitisation of ion radiotherapy: a review. Physica Medica 2018, 47, 121-128, DOI: 10.1016/j.ejmp.2018.03.004.

(8)Vankayala, R.; Kuo, C.-L.; Nuthalapati, K.; Chiang, C.-S.; Hwang, K.-C. Nucleus-Targeting Gold Nanoclusters for Simultaneous In Vivo Fluorescence Imaging, Gene Delivery, and NIRLight Activated Photodynamic Therapy. Advanced Functional Materials 2015, 25, 5934-5945. DOI: $10.1002 /$ adfm.201502650.

(9) Cleveland, C. L.; Landman, U.; Schaaff, T. G.; Shafigullin, M. N.; Stephens, P. W.; Whetten, R. L. Structural Evolution of Smaller Gold Nanocrystals: The Truncated Decahedral Motif. Phys. Rev. Lett. 1997, 79, 1873. DOI: 10.1103/PhysRevLett.79.1873.

(10) Koga, K.; Takeo, H.; Ikeda, T.; Ohshima, K.-I. In situ grazing-incidence x-ray-diffraction and electron-microscopic studies of small gold clusters. Phys. Rev. B 1998, 57, 4053. DOI: 10.1103/PhysRevB .57.4053. 
(11) Koga, K.; Ikeshoji, T.; Sugawara, K.-I. Size- and Temperature-Dependent Structural Transitions in Gold Nanoparticles. Phys. Rev. Lett. 2004, 92, 115507. DOI: 10.1103/PhysRevLett.92.115507.

(12) Li, Z. Y.; Young, N. P.; Di Vece, M.; Palomba, S.; Palmer, R. E.; Bleloch, A. L.; Curley, B. C.; Johnston, R. L.; Jiang, J.; Yuan, J. Three-dimensional atomic-scale structure of size-selected gold nanoclusters. Nature 2008, 451, 46-48. DOI: 10.1038/nature06470.

(13) Foster, D. M.; Ferrando, R.; Palmer, R. E. Experimental determination of the energy difference between competing isomers of deposited, size-selected gold nanoclusters. Nature Communications 2018, 9, 1323. DOI: 10.1038/s41467-018-03794-9.

(14) Plant, S. R.; Cao, L.; Palmer, R. E. Atomic Structure Control of Size-Selected Gold Nanoclusters during Formation. J. Am. Chem. Soc. 2014, 136, 7559-7562. DOI: 10.1021/ja502769v.

(15) Butterworth, K. T.; Coulter, J. A.; Jain, S.; Forker, J.; McMahon, S. J.; Schettino, G.; Prise, K. M.; Currell, F. J.; Hirst D. G. Evaluation of cytotoxicity and radiation enhancement using 1.9 nm gold particles: potential application for cancer therapy. Nanotechnology 2010, 21 (29), 295101. DOI: $10.1088 / 0957-4484 / 21 / 29 / 295101$.

(16) Jain, S.; Coulter, J. A.; Butterworth, K. T.; Hounsell, A. R.; McMahon, S. J.; Hyland, W. B.; Muir, M. F.; Dickson, G. R.; Prise, K. M.; Currell, F. J.; Hirst, D. G.; O’Sullivan, J. M. Gold nanoparticle cellular uptake, toxicity and radiosensitisation in hypoxic conditions. Radiotherapy and Oncology 2014, 110 (2), 342-347. DOI: 10.1016/j.radonc.2013.12.013. 
(17) Gervais, B.; Beuve, M.; Olivera, G.H.; Galassi, M.E.; Rivarola, R.D. Production of HO2 and $\mathrm{O} 2$ by multiple ionization in water radiolysis by swift carbon ions. Chem. Phys. Lett. 2005, 410 (4-6), 330-334, DOI: 10.1016/j.cplett.2005.05.057.

(18) Gervais, B.; Beuve, M.; Olivera, G.H.; Galassi, M.E. Numerical simulation of multiple ionization and high LET effects in liquid water radiolysis. Radiat. Phys. Chem. 2006, 75 (4), 493513, DOI: 10.1016/j.radphyschem.2005.09.015.

(19) Meng, S.; Wang, E. G.; Gao, S. Water adsorption on metal surfaces: a general picture from density functional theory studies. Phys. Rev. $B$ 2004, 69, 195404-1,13, DOI: 10.1103/PhysRevB.69.195404.

(20) Phatak, A. A.; Delglass, W. N.; Ribeiro, F. H.; Schneider, W. F. Density functional theory comparison of water dissociation steps on $\mathrm{Cu}, \mathrm{Au}, \mathrm{Ni}, \mathrm{Pd}$ and Pt. J. Phys. Chem. C 2009, 113, 7269-7276, DOI: 10.1021/jp810216b.

(21) Nadler, R.; Sanz, J. F. Effect of dispersion correction on the Au (1 11 1)-H2O interface: a first-principles study. J. Chem. Phys. 2012, 137(11), 114709. DOI: 10.1063/1.4752235.

(22) Carrasco, J.; Klimes, J.; Michaelides, A. The role of van der Waals forces in water adsorption on metals. J. Chem. Phys. 2013, 138, 024708-1,9, DOI: 10.1063/1.4773901.

(23) Berg, A.; Peter, C.; Johnston, K. Evaluation and Optimization of Interface Force Fields for Water on Gold Surfaces. J. Chem. Theory Comput. 2017, 13(11), 5610-5623. DOI: 10.1021/acs.jctc.7b00612.

(24) Lin, X.; Groß, A. First-principles study of the water structure on flat and stepped gold surfaces. Surf. Sci. 2012, 606, 886-891. DOI: 10.1016/j.susc.2011.12.01. 
(25) Xue, Y. Water monomer interaction with gold nanoclusters from van der Waals density functional theory. J. Chem. Phys. 2012, 136(2), 024702, DOI: 10.1063/1.3675494.

(26) de Morais, R. F.; Kerber, T.; Calle-Vallejo, F.; Sautet, P.; Loffreda, D. Capturing solvation effects at a liquid/nanoparticle interface by Ab Initio molecular dynamics: Pt201 immersed in water. Small 2016, 12(38), 5312-5319. DOI: 10.1002/smll.201601307.

(27) Ju, S.-P. A molecular dynamics simulation of the adsorption of water molecules surrounding an Au nanoparticle. J. Chem. Phys. 2005, 122, 094718-1,6, DOI: 10.1063/1.1854132.

(28) Chang, C. I.; Lee, W. J.; Young, T. F.; Ju, S. P.; Chang, C. W.; Chen, H. L.; Chang, J. G. Adsorption mechanism of water molecules surrounding Au nanoparticles of different sizes. $J$. Chem. Phys. 2008, 128(15), 154703. DOI: 10.1063/1.2897931.

(29) Häberlen, O. D.; Chung, S.-C.; Stener, M.; Rösch, N. From clusters to bulk: a relativistic density functional investigation on a series of gold clusters Au n, n=6,..,147. J. Chem. Phys. 1997, 106, 5189-5201. DOI: 10.1063/1.473518.

(30) Ferrando, R.; Fortunelli, A.; Rossi, G. Quantum effects on the structure of pure and binary metallic nanoclusters. Phys. Rev. B 2005, 72, 085449-1,9. DOI: 10.1103/PhysRevB.72.085449.

(31) Logsdail, A. J.; Li, Z. Y.; Johnston, R. L. Faceting preferences for AuN and PdN nanoclusters with high-symmetry motifs. Phys. Chem. Chem. Phys. 2013, 15, 8392-8400. DOI: 10.1039/c3cp50978h.

(32) Li, H.; Li, L.; Pedersen, A.; Gao, Y.; Khetrapal, N.; Jónsson, H.; Zeng, X. C. Magic-number gold nanoclusters with diameters from 1 to $3.5 \mathrm{~nm}$ : Relative stability and catalytic activity for CO oxidation. Nano Lett. 2015, 15(1), 682-688. DOI: 10.1021/n1504192u. 
(33) Tarrat, N.; Rapacioli, M.; Cuny , J.; Morillo, J.; Heully, J.-L.; Spiegelman, F. Global optimization of neutral and charged 20- and 55-atom silver and gold clusters at the DFTB level. Computational and Theoretical Chemistry 2017, 1107, 102-114. DOI: 10.1016/j.comptc.2017.01.022.

(34) Tarrat, N.; Rapacioli, M.; Spiegelman F. Au147 nanoparticles: ordered or amorphous? J. Chem. Phys. 2018, 148, 204308. DOI: 10.1063/1.5021785.

(35) Zhu, B.; Xu, Z.; Wang, C.; Gao, Y. Shape evolution of metal nanoparticles in water vapor environment. Nano Lett. 2016, 16(4), 2628-2632. DOI: 10.1021/acs.nanolett.6b00254.

(36) Baletto, F.; Ferrando, R.; Fortunelli, A.; Montalenti, F.; Mottet, C. Crossover among structural motifs in transition and noble-metal clusters. J. Chem. Phys. 2002, 116(9), 3856-3863. DOI: $10.1063 / 1.1448484$.

(37) Baletto, F.; Ferrando, R. Structural properties of nanoclusters: Energetic, thermodynamic, and kinetic effects. Rev. Mod. Phys. 2005, 77(1), 371. DOI: 10.1103/RevModPhys.77.371.

(38) Rahm, J. M.; Erhart, P. Beyond Magic Numbers: Atomic Scale Equilibrium Nanoparticle Shapes for Any Size. Nano Lett. 2017, 17(9), 5775-5781. DOI: 10.1021/acs.nanolett.7b02761.

(39) Wang, B.; Liu, M.; Wang, Y.; Chen, X. Structures and energetics of silver and gold nanoparticles. J. Phys. Chem. C. 2011, 115(23), 11374-11381. DOI: 10.1021/jp201023x.

(40) Levitt, M.; Hirschberg, M.; Sharon, R.; Laidig, K. E.; Daggett, V. Calibration and testing of a water model for simulation of the molecular dynamics of proteins and nucleic acids in solution J. Phys. Chem. B 1997, 101, 5051-5061. DOI: 10.1021/jp964020s. 
(41) Santra, B.; Michaelides, A.; Scheffler, M. Coupled cluster benchmarks of water monomers and dimers extracted from density-functional theory liquid water: the importance of monomer deformations J. Chem. Phys. 2009, 131, 124509-1,9. DOI: 10.1063/1.3236840.

(42) Soper, A. K.; Bruni, F.; Ricci, M. A. Site-site pair correlation functions of water from 25 to $400{ }^{\circ} \mathrm{C}$ : Revised analysis of new and old diffraction data. J. Chem. Phys. 1997, 106, 247. DOI: 10.1063/1.473030.

(43) Guardia, E.; Skarmoutsos, I.; Masia, M. Hydrogen Bonding and Related Properties in Liquid Water: A Car-Parrinello Molecular Dynamics Simulation Study. J. Phys. Chem. B 2015, 119 (29), 8926-8938. DOI: 10.1021/jp507196q.

(44) Kresse, G.; Hafner, J. Ab initio Molecular Dynamics for Liquid Metals. Phys. Rev. B 1993, 47, 558. DOI: 10.1103/PhysRevB.47.558.

(45) Kresse, G.; Furthmüller, J. Efficiency of Ab-initio Total Energy Calculations for Metals and Semiconductors Using a Plane-wave Basis Set. Comput. Mat. Sci. 1996, 6, 15-50. DOI: 10.1016/0927-0256(96)00008-0.

(46) Kresse, G.; Furthmüller, J. Efficient Iterative Schemes for Ab-initio Total-energy Calculations Using a Plane-wave Basis Set. Phys. Rev. B 1996, 54, 11169. DOI: 10.1103/PhysRevB.54.11169.

(47) Perdew, J. P.; Burke, K.; Ernzerhof, M. Generalized Gradient Approximation Made Simple. Phys. Rev. Lett. 1996, 77, 3865. DOI: 10.1103/PhysRevLett.77.3865. 
(48) Grimme, S.; Antony, J.; Ehrlich, S.; Krieg, H. A consistent and accurate ab initio parametrization of density functional dispersion correction (DFT-D) for the 94 elements H-Pu. $J$. Chem. Phys. 2010, 132, 154104. DOI: 10.1063/1.3382344.

(49) Kresse, G.; Joubert, D. From Ultrasoft Pseudopotentials to the Projector Augmented-wave Method. Phys. Rev.B 1999, 59, 1758. DOI: 10.1103/PhysRevB.59.1758. 
$\Delta \mathrm{E}_{\mathrm{coh}}(\mathrm{NP})(\mathrm{eV})$

-2.6 The tournal of Physical Chemistrypleegeer6 of 30

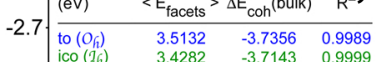

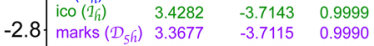

\begin{tabular}{lllll} 
2.9. & cubo $\left(O_{h}\right)$ & 3.4377 & -3.7087 & 0.9996 \\
\hline
\end{tabular}

-3.0
-3.

3

$-3.1$

$-32$

923780604428

309

$561 \quad{ }_{509}^{309} 287$

$-36.923835$

181

55

55

38

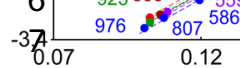

${ }_{5}^{8} e_{\text {exc }}(\mathrm{NP})(\mathrm{eV})$

0.17

$N^{-1 / 3} \quad 0.22$

0.27

0.32

(b)

3.490

$3.4 d .188^{55}$

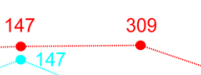

$\Delta E_{e x c}(N P)=\left(E_{\text {tot }}(N P)-N \cdot E_{\text {tot }}(\right.$ bulk $\left.)\right) / N^{2 / 3}$

3.3. $\frac{255}{54}$

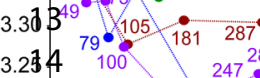

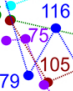

\section{9}

561

3.225

$146_{176}^{\circ} \cdot{ }_{201}^{24}$

$282 \quad 383$

3.196

$$
201 \mathrm{P}_{314}
$$

19

$100 \quad 200 \quad 300$

$\because \div$

$433 \quad \because 609$ deca

561

cubo

- 923

428

10

$\mathrm{N}$ 


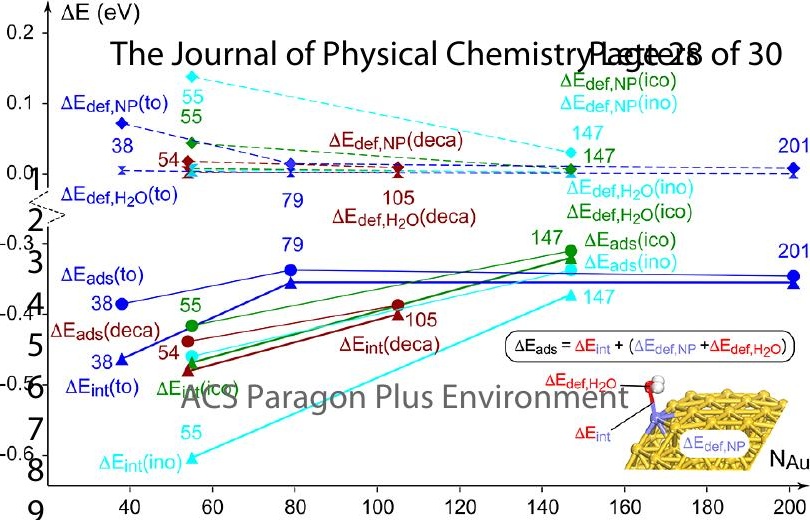




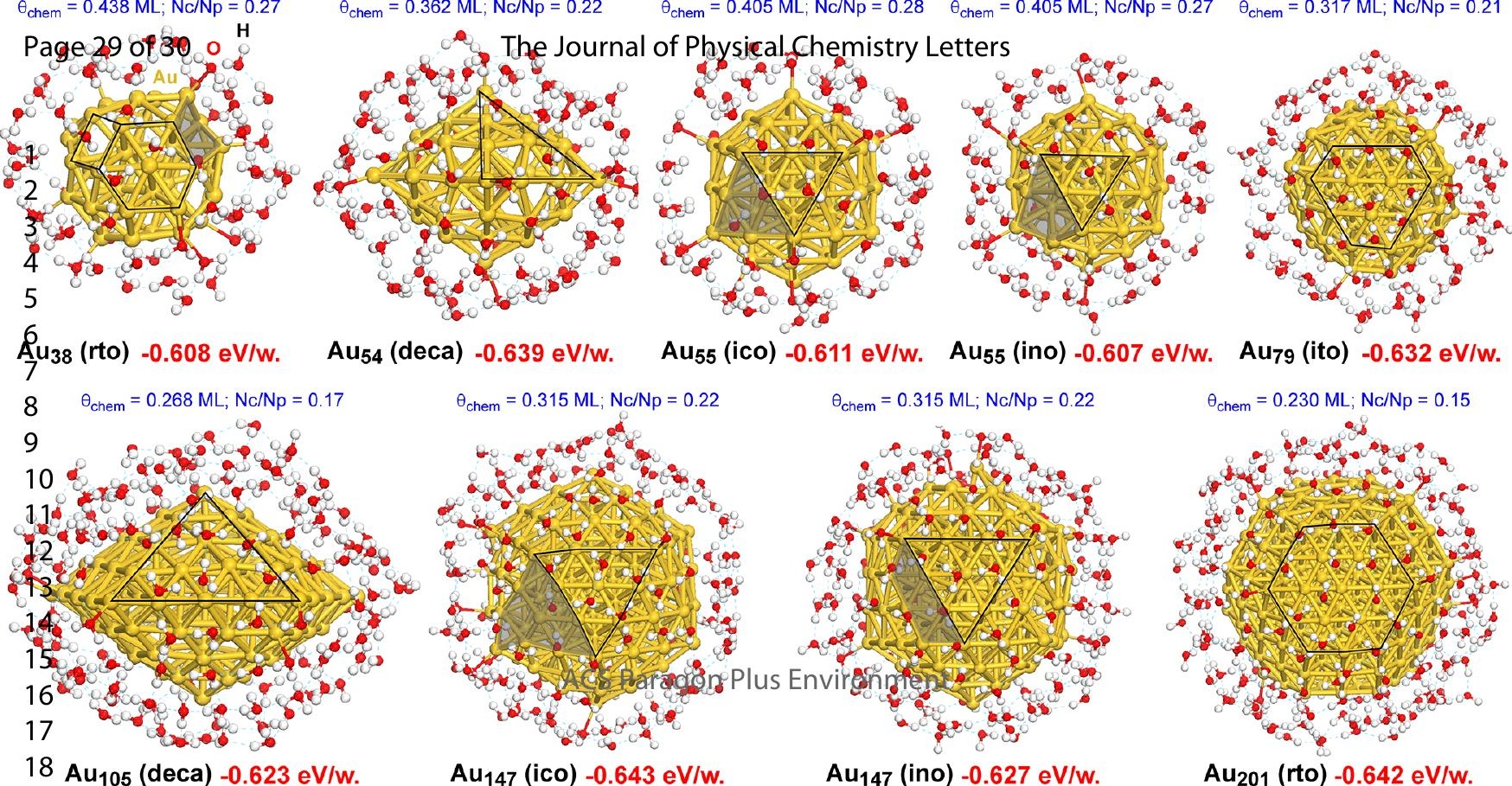




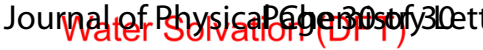
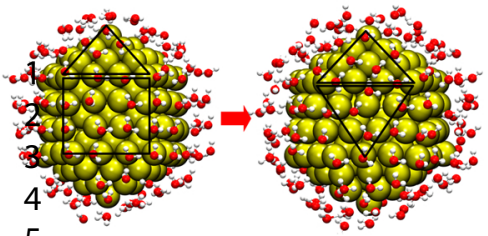

5
ACC SAdinagon
ifo-decahedron

Plus EnviAblnment icosahedron

7 metastable)

(stable)

8 